\title{
Rethinking an Algorithm: The Utility of the Techinicium 99 m Labeled Red Cell (RBC) Scanning for Lower Gastrointestinal Bleeding (LGIB)
}

\author{
Shaffer R. S. Mok ${ }^{1}$, Chijioke Ojiako ${ }^{2}$, Ankur Kalra ${ }^{1}$, Mithil Gajera ${ }^{3}$, Sri Sujanthy Rajaram ${ }^{3}$ \\ ${ }^{1}$ Division of Internal Medicine, Department of Internal Medicine, Cooper University Hospital, Robert Wood Johnson Medical School, \\ University of Medicine and Dentistry of New Jersey, Camden, USA; ${ }^{2}$ Mulica Hill Medical Associates, Mullica Hill, USA; ${ }^{3}$ Division \\ of Critical Care Medicine, Department of Internal Medicine, Cooper University Hospital, Robert Wood Johnson Medical School, \\ University of Medicine and Dentistry of New Jersey, Camden, USA. \\ Email: mok-shaffer@cooperhealth.edu
}

Received December $9^{\text {th }}, 2011$; revised January $18^{\text {th }}, 2012$; accepted February $26^{\text {th }}, 2012$

\begin{abstract}
Purpose: Technetium $99 \mathrm{~m}$ (99 m-Tc) labeled scan is often done to localize bleeding to facilitate treatment. No level 1 or 2 data supports this approach. The aim of this study was to determine the correlation between site of bleeding by nuclear scan and findings at surgery, angiogram or colonoscopy. Methods: Records of patients admitted to Cooper University Hospital from January 2001-December 2005 with LGIB who had 99mTc scan were analyzed. Results: 164 of 170 patients were eligible to be evaluated. There were 45 positive (27.5\%) and 119 negative scans (72.5\%). 21 of 45 patients with positive scans had angiography. 7 patients (33.3\%) had positive and 14 (66.6\%) negative angiograms. In 6 patients (85.7\%) with (+) angiograms, there was correlation on the area of bleed as seen on the $99 \mathrm{~m}$-Tc scan $(\mathrm{p}=$ 0.125). 20 patients, in the positive scan group, required surgery. In $15(75 \%)$ the findings at surgery correlated with the scan result $(\mathrm{p}=0.04)$. 31 patients $(68.8 \%)$ with positive scan had colonoscopy. There was correlation in 27 patients $(87.0 \%)(\mathrm{p}<0.001)$. The patients with $(+)$ scan received a total of $372(8.2$ per patient) transfusions of packed red blood cells (PRBC) compared to 333 (2.7 per patients) transfusions in patients with (-) scans. Surgeons documented in 7 patients that the result of scan influenced surgery. Patients with $(+)$ and $(-)$ scans had similar rates of colonoscopy $(73.35 \%$ vs $76.4 \%$ ), hospital length of stay (14.3 vs 12.10 days), while mortality rate was ( $8.8 \%$ vs $6.72 \%)$ respectively, Conclusion: 99 m-Tc scan has low yield in the evaluation of LGIB. However when positive, they tend to correlate with findings at angiogram, surgery and colonoscopy.
\end{abstract}

Keywords: Technicium 99 m; Lower Gastrointestinal Bleed; LGIB; Tc-99 m

\section{Introduction}

Gastrointestinal Bleeding (GIB) is a major source of morbidity and mortality in the Unites States, encompassing 800,000 discharge diagnoses yearly. Of these known diagnoses lower GIB (LGIB), as defined as bleeding distal to the ligament of Trietz, included 20 to 27 cases per 100,000 admissions in the United States [1]. To better understand this condition, a diagnostic algorithm was created to proceed with an accurate and cost-effective method for diagnosis [2]. Patients that are hemodynamically unstable undergo upper endoscopy followed by additional diagnostic means if no source of bleeding is located. One such diagnostic modality is the $99 \mathrm{~m}$-Technitium (99 mTc) labeled RBC scan, however no level 1 or 2 data exists to support this diagnostic modality in the setting of acute LGIB.

When examining the existing data accuracy rates were reported for the $99 \mathrm{~m}$-Tc labeled RBC scan, ranging from $0 \%$ to $96 \%$ in localizing the site of bleeding [3-5]. These results were compared with angiography, endoscopy or surgical means [6]. What is more concerning are the rates for which the $99 \mathrm{~m}$-Tc scan can direct surgical intervenetion. Hunter, et al. reported $42 \%$ of subjects who had "positive" 99 m-Tc scanning underwent unnecessary surgical procedures [7]. Data by Voeller, et al. demonstrated zero of the "positive" tagged RBC scans guiding surgical intervention and of the 18 subjects who required surgical intervention 11 had negative $99 \mathrm{~m}$-Tc scanning [8]. With such variable results and the potential of subjecting patients to unnecessary surgical procedures, the clinical utility of the $99 \mathrm{~m}$-Tc labeled RBC scanning test is in question. The goal of this study was to determine; the accuracy rate of the $99 \mathrm{~m}$-Tc scan, correlation between results of scan and findings at the time of surgery, endo- 
scopy and angiography, influence of the scan on surgical intervention, blood transfusion requirements, mortality and hospital length of stay (LOS).

\section{Methods}

\subsection{Study Design}

This retrospective case series study was approved by the IRB at Cooper University Hospital and protocol was consistent with all ethical guideline set forth by the declaration of Helsinki. Eligible subject data was screened by admission diagnosis of acute lower gastrointestinal bleed (LGIB) and underwent 99 m-Technitium (99 m-Tc) Scanning. All subjects were admitted to Cooper University Hospital/The University of Medicine and Dentistry of New Jersey-Robert Wood Johnson Medical School-Camden from January 2001 to December 2005.

This study population was examined first for age and sex. After this distinction was made, the sample was then analyzed for the following: 1) Accuracy Rate of $99 \mathrm{~m}$-Tc scan; 2) Correlation between site of bleeding as detected by $99 \mathrm{~m}$-Tc scan and findings at the time of surgery; 3 ) The influence of the $99 \mathrm{~m}$-Tc scan on the type of surgery; 4) Correlation between findings at the time of scan and findings at time of angiogram and colonoscopy; 5) Blood transfusion requirements (units of packed red blood cells (PRBC)); 6) Mortality and hospital length of stay (days).

\subsection{Study Measurements}

The sample population underwent $99 \mathrm{~m}$-Technetium tagged RBC scanning and followed all guidelines set forth by The Society of Nuclear Medicine [9]. Intravenous (IV) access was first obtained utilizing standard techniques. Subjects were then injected with 20 to $30 \mathrm{mCi}$ (750 to $1100 \mathrm{MBq}$ ) of an isotope containing $99 \mathrm{~m}$-Technetium RBCs. After injection, the technician waited 15 to 20 minutes for the isotope to circulate. At this time, the subject population underwent a series of gamma-camera nuclear scan guided imaging at $1 \mathrm{~min}$ image duration, taken at 5 minute intervals in cine mode for 90 minutes to localize extravagation of the isotope. Both anterior-posterior and oblique imaging was obtained to differentiate imaging form the bladder. The rectal glove was also imaged at this time to localize sources of rectal bleeding. If no tracer was extravagated by 90 minutes, the study was terminated. All imaging was scrutinized by board certified Radiologists accustomed to analyzing nuclear tagged RBC scans.

Angiographic techniques involved the department of Surgery in conjunction with Interventional Radiology and were in accordance with guidelines set forth by the American College of Cardiology/American Heart Association [10]. Finally exploratory laparotomy was performed by the Department of Surgery, specific guideline could not be elicited.

\subsection{Statistical Methods}

Several different analyses were utilized to determine the results of this study. A sample size of 150 was determined to obtain adequate power for this study. Ascertaining 40 from the positive group and 110 from the negative group achieve $86 \%$ power for detecting a difference with $95 \%$ confidence interval. Categorical data (e.g. mortality) was compared using Fischer's exact test. A chi-square was completed for each of these variables, with the $\mathrm{p}$ value, $95 \%$ CI, being calculated by standard means. Continuous variables (e.g. Length of hospital stay) were compared using the two-tailed student's t-test. Multivariate analyses were used to examine the elements of outcome.

\section{Results}

\subsection{Sample Population}

Of the total sample population of 170 subjects, 164 subjects were included in the study. The exclusion of 6 subjects occurred due to incomplete medical records. Of the 164 subjects $(\mathrm{n}=164), 67(40.9 \%)$ were male, $97(59.1 \%)$ were female. The mean age of the sample population was determined as 70.18 years of age $(+/-12.36$, standard deviation (SD)). The mean age of the positive RBC scan subgroup showed no statistically significant difference when compared to the negative subgroup $(p=0.85)($ Table 1).

Table 1. Incidence of gender, age and diagnostic test results in sample population.

Positive $99 \mathrm{~m}$-Tc Negative $99 \mathrm{~m}$-Tc Labeled RBC Scan Labeled RBC Scan

Sample Population

$\begin{array}{lll}\text { Male } & 17 & 50 \\ \text { Female } & 28 & 69 \\ \text { TOTAL } & 45 & 119 \\ \text { Mean Age (years) } & 69.9 & 70.3 \\ \text { Diagnostic Testing } & & \\ \text { Angiography } & 7(15.56) & 1(0.84) \\ \text { Positive (incidence in percent) } & 14(31.11) & 7(5.88) \\ \text { Angiography Negative } & 24(53.33) & 111(93.28) \\ \text { No Angiography } & 32(71.11) & 87(73.11) \\ \text { Colonoscopy Positive } & 1(2.22) & 5(4.20) \\ \text { Colonoscopy Negative } & 12(26.67) & 27(22.69) \\ \text { No Colonoscopy } & 20(44.44) & 8(6.72) \\ \text { Surgery Positive } & 0 & 0 \\ \text { Surgery Negative } & 35(55.56) & 111(93.28) \\ \text { No Surgery } & & \end{array}$




\subsection{Subgroup Analysis}

Of the 164 subjects, 45 (27.5\%) of the sample population had a positive test, while 119 (72.5\%) of the total sample size had negative $99 \mathrm{~m}$-Tc labeled RBC scans. Within the 45 subjects who had positive $99 \mathrm{~m}$-Tc Scanning, 21 (46.67\%) underwent angiography, 20 (44.44\%) subjects required surgery and 31 (68.89\%) subjects required colonoscopy. The rates of colonoscopy were similar between the positive RBC scan group (73.35\%) when compared with the negative group (76.4\%). Among the 21 subjects who underwent angiography, 7 (33.33\%) subjects had positive angiograms and 14 (66.67\%) subjects had negative angiograms (Table 1).

The sensitivities, specificities, positive predictive values (PPV) and negative predictive values of the $99 \mathrm{~m}-\mathrm{Tc}$ Scan when compared to angiography results, colonoscopy results and surgical results can be summarized in Table 3. The accuracy rate of the tagged RBC scan was reported as $35.71 \%$ when compared to surgery, $55.11 \%$ versus colonoscopy and $77.08 \%$ when compared to angiography.

In 6 of the 7 subjects, who had positive 99 m-Tc labeled RBC scans and positive angiography, there was correlation found between the angiographic findings and the results of the tagged RBC scan. These findings were not statistically significant $(\mathrm{p}=0.125)$. Within the surgical subgroup 15 of the 20 subjects, that required surgery and had positive $99 \mathrm{~m}$-Tc labeled RBC scans, had results that correlated between the scan and surgical findings ( $p$ $=0.04)$. Attending surgeons in 7 (46.67\%) of subjects who underwent surgery reported that results of the $99 \mathrm{~m}-\mathrm{Tc}$ scan "influenced" their surgery. The colonoscopy subgroup showed correlation in 27 subjects (87\%, p < 0.001), also statistically significant.

When examining the transfusion requirements of the groups with positive $99 \mathrm{~m}$-Tc labeled RBC scanning with the negative group, the positive group was transfused 372 units of PRBC (8.3 per subject), compared to 333 units of PRBC in the negative group (2.8 units per subject) ( $p$ $<0.001)$. Subjects in the $99 \mathrm{~m}$-Tc labeled RBC scan positive group demonstrated a mean hospital LOS of 14.4 days when compared with 10.3 days of the negative subgroup ( $p=0.09$ ). Mortality rates were reported as $8.8 \%$ and $6.7 \%$ among the positive and negative subgroups respectively ( $\mathrm{p}=0.85$ ) (Table 2 ).

\section{Discussion}

The diagnostic approach to LGIB has been defined in an algorithmic approach [2]. Within this approach, is the subdivision of subjects that are hemodynamically unstable, defined by hypotension, tachycardia or postural changes positive in blood pressure. The approach to these patients required upper endoscopy as the initial diagnostic step. If
Table 2. Transfusion requirements, hospital length of stay and mortality of sample population.

\begin{tabular}{llll}
\hline & $\begin{array}{l}\text { Positive 99 m-Tc } \\
\text { Labeled RBC Scan }\end{array}$ & $\begin{array}{l}\text { Negative 99 m-Tc } \\
\text { Labeled RBC Scan }\end{array}$ & p Value \\
\hline $\begin{array}{l}\text { Transfusion } \\
\text { Requirements }\end{array}$ & & & \\
$\begin{array}{l}\text { Total Number of } \\
\text { PRBCs (units) }\end{array}$ & 372 & 333 & $<0.001$ \\
$\begin{array}{l}\text { PRBC/subject } \\
\text { (units/subject) }\end{array}$ & 8.3 & 2.8 & $<0.001$ \\
$\begin{array}{l}\text { Hospital Length of } \\
\text { Stay (days) }\end{array}$ & 14.4 & 10.3 & 0.09 \\
\begin{tabular}{l} 
Mortality (\%) \\
\hline
\end{tabular} & 8.8 & 6.7 & 0.85 \\
\hline
\end{tabular}

Table 3. Diagnostic Parameters of 99 m-Technitium Labeled RBC Scan when compared with various diagnostic modalities.

\begin{tabular}{|c|c|c|c|c|c|}
\hline & $\begin{array}{l}\text { Sensitivity } \\
\text { (\%) }\end{array}$ & $\begin{array}{l}\text { Specificity } \\
(\%)\end{array}$ & $\begin{array}{l}\text { Positive } \\
\text { Predictive } \\
\text { Value (\%) }\end{array}$ & $\begin{array}{l}\text { Negative } \\
\text { Predictive } \\
\text { Value (\%) }\end{array}$ & $\begin{array}{l}\text { Accuracy } \\
\text { Rate (\%) }\end{array}$ \\
\hline $\begin{array}{l}99 \text { mTc Labelec } \\
\text { RBC Scan } \\
\text { versus } \\
\text { Angiography }\end{array}$ & d 87.5 & 33.33 & 33.33 & 87.5 & 77.08 \\
\hline $\begin{array}{l}99 \text { mTc Labelec } \\
\text { RBC Scan } \\
\text { versus } \\
\text { Colonoscopy }\end{array}$ & d 26.89 & 83.33 & 96.97 & 5.43 & 55.11 \\
\hline $\begin{array}{l}99 \text { mTc Labelec } \\
\text { RBC Scan } \\
\text { versus Surgery }\end{array}$ & d 71.43 & 0 & 100 & 0 & 35.71 \\
\hline
\end{tabular}

bleeding persisted despite endoscopic intervention, the recommendation is to proceed additional diagnostic modalities one of which is the $99 \mathrm{~m}$-Tc labeled RBC scan. Though included in the diagnostic approach for LGIB, variable data existed in our literature search to support utilization of the $99 \mathrm{~m}$-Tc RBC scan.

The accuracy of the scan is limited by the method that $99 \mathrm{~m}$-Tecnitium is instilled into the patient. By utilizing the sulfur colloid approach (SC), studies suggested that sources of bleeding could be identified for patients bleeding as low as 0.05 to 0.1 milliliter/minute ( $\mathrm{mL} / \mathrm{min})$ [11]. Future studies determined bleeding rates as low as 0.04 $\mathrm{mL} / \mathrm{min}$ by utilizing instillation of $99 \mathrm{~m}$-Tc tagged RBC directly into the patient [12]. Indeed this modality appeared promising, however when put to the test, results were less hopeful.

Our study determined the sensitivity, specificity, etc., for the 99 m-Tc labeled RBC scan when compared with various diagnostic methods deemed more "accurate". The results showed much variability of the RBC scan when compared to various diagnostic modalities (Table 3). 
Additionally, the accuracy rate of the $99 \mathrm{~m}$-Tc scan ranged from $35.71 \%$ to $77.08 \%$ when compared with various other investigative techniques. These results are likely due to the varying diagnostic parameters of the other, more accurate, diagnostic tests.

The 99 m-Tc RBC scan likely was found to have a higher accuracy rate when compared to angiography, as the reported sensitivity of angiography ranged from $30 \%$ 47\% [13-15]. Similarly, the accuracy rate of the RBC scan when compared to colonoscopy was likely due to the ability of endoscopy to detect $74 \%-82 \%$ of source of GIB, which is higher than that of angiography [16]. Finally, the accuracy rate of the $99 \mathrm{~m}$-Tc scan was lowest when compared to surgery, as surgery allows direct visualization of the patient in questions anatomy and is therefore the most accurate diagnostic approach.

A large review of the data on radiolabeled RBC scanning, by Zucker et al., yielded the percentages of "correct" scans from zero to $96 \%$ [6]. The confirmation of the 99 m-Tc labeled RBC scan's results occurred through endoscopic, angiographic and surgical means. Our study utilized similar means to correlate the labeled RBC scans. In terms of correlation with angiography, the results of our study determined that one third of the subjects with a "positive" tagged RBC scan also had angiographic evidence of bleeding. Furthermore, six of those seven had correlation with the location of bleeding, though not statistically significant $(p=0.125)$. These results are likely explained by the variability of both diagnostic tests for determining location of GIB, as stated above.

In contrast, statistical significance was found when the $99 \mathrm{~m}$-Tc scans were utilized to localize bleeding in comparison to surgery $(\mathrm{p}=0.04)$ and colonoscopy $(\mathrm{p}<0.001)$ (Table 1). In comparison to data from Hunter et al., the localization error was comparable when $99 \mathrm{~m}$-Tc scanning was weighed against surgical means in approximately $25 \%$ of cases [7]. The danger of assuming correlation between the $99 \mathrm{~m}$-Tc scan and surgery is the potential to perform surgery when unnecessary. Orecchia, et al., found a correlation of $94 \%$ among his sample population, while Voeller demonstrated failure of localization in $85 \%$ of his sample population [8,17]. The data is conflicting, however our data does show statistical correlation between $99 \mathrm{~m}$-Tc scanning and surgery/colonoscopy. Despite this signifycance, the surgeons of seven subjects were "influenced" by $99 \mathrm{~m}$-Tc RBC scans. This small number alluded to the need for imaging more advanced than the RBC scan, when pursuing surgical intervention in LGIB. Additionally, if the diagnostic yield is comparable, perhaps RBC scan is an unnecessary and costly step in the diagnostic work up of LGIB. Arguments for the contrary would offer contention to the invasiveness of angiography, endoscopy and surgical means.
Another interesting result of this study is the relationship between the subjects with a positive $99 \mathrm{~m}$-Tc scan and those with a "negative" scan. As defined in Table 2, the subjects who had a positive $99 \mathrm{~m}$-Tc labeled RBC scan, required a statistically significant amount of PRBC per subject when compared with the subjects with a negative scan. When examining hospital LOS, the positive RBC scan subgroup had an additional 4.1 days when compared with the negative subgroup, though not statistically significant $(p=0.09)$. Finally, there was no significant difference calculated between the all cause mortalities of the scan positive and negative subgroups $(p=0.85)$. To our knowledge, no literature existed to correlate transfusion demands, LOS or all cause mortality with $99 \mathrm{~m}$-Tc labeled RBC scanning. The results of this study appeared to demonstrate the transfusions demands increased with a positive test, but no statistically significant difference existed in LOS or mortality between the two subgroups.

Potential limiting factors included the retrospective nature of this study, which could be prevented with a future prospective study. Additionally, the sample size of subjects who underwent further diagnostic investigation was small and may have limited data extrapolation. The presence of small sample size was consistent with data found during our literature search. Future study with a larger cohort may expand knowledge of the true clinical utility of this test for LGIB. Yet despite these potential limitations, the results of this study call into question the diagnostic utility of 99-Tc scanning when confirmatory testing exists with greater accuracy. Though less invasive, the potential for localization error of $25 \%$ may lead to a high number of unnecessary surgeries in patients with LGIB.

\section{Conclusion}

The $99 \mathrm{~m}$-Tc labeled RBC scan is a diagnostic study for LGIB with relatively low diagnostic accuracy rates. However when the study was positive, the location of LGIB appeared to correlate with the results of angiography, colonoscopy and surgery. There was a statistically significant increase in the units of PRBC per subject in the subgroup with a positive RBC scan when compared to the subgroup with a negative scan. Finally, there was no statistical significance between the hospital LOS and mortality of the positive versus negative subgroups.

\section{Acknowledgements}

The creators of this study would like to acknoledge the faculty and staff, as well as the members of the medical records departement at Cooper University hospital. 


\section{REFERENCES}

[1] J. J. Farrell and L. S. Friedman, “Gastrointestinal Bleeding in the Elderly," Gastroenterology Clinics of North America, Vol. 30, No. 2, 2001, pp. 377-407. doi:10.1016/S0889-8553(05)70187-4

[2] A. S. Fauci, E. Braunwald, D. L. Kasper, et al., "Harrison's Principles of Internal Meidcine,” 17th Edition, McGrawHill Companies, Inc., New York, 2008.

[3] T. E. Garofalo and R. A. Abdu, “Accuracy and Efficacy of Nuclear Scintigraphy for the Detection of Gastrointestinal Bleeding," Archives of Surgery, Vol. 132, No. 2, 1997, pp. 196-199. doi:10.1001/archsurg.1997.01430260094020

[4] M. S. Suzman, M. Talmor, et al., “Accurate Localization and Surgical Management of Active Lower Gastrointestinal Hemorrhage with Technetium-Labeled Erythrocyte Scintigraphy,” Annals of Surgery, Vol. 226, No. 1, 1997, pp. 112-113.

[5] R. Levy, W. Barto and J. Gani, "Retrospective Study of the Utility of Nuclear Scintigraphic-Labeled Red Cell Scanning for Lower Gastrointestinal Bleeding," Annals of Surgery, Vol. 73, 2003, pp. 205-220. doi:10.1046/j.1445-1433.2002.02567.x

[6] D. A. Zukerman, T. P. Bocchini and E. H. Birnbaum, "Massive Hemorrhage in the Lower Gastrointestinal Tract in Adults: Diagnostic Imaging and Intervention,” American Journal of Roentgenology, Vol. 161, No. 4, 1993, pp. 703-711.

[7] J. M. Hunter and M. E. Pezim, "Limited Value of Technetium 99 m-Labeled Red Cell Scintigraphy in Localization of Lower Gastrointestinal Bleeding," American Journal of Surgery, Vol. 159, No. 5, 1990, pp. 504506. doi:10.1016/S0002-9610(05)81256-5

[8] G. R. Voeller, G. Bunch and L. G. Britt, "Use of Technetium-Labeled Red Blood Cell Scintigraphy in the Detection and Management of Gastrointestinal Hemorrhage,” Surgery, Vol. 110, No. 4, 1991, pp. 799-804.

[9] P. V. Ford, S. P. Bartold, D. M. Fink-Bennett, et al., "Procedure Guideline for Gastrointestinal Bleeding and Meckel's Diverticulum Scintigraphy. Society of Nuclear Medicine,” Journal of Nuclear Medicine, Vol. 40, No. 7, 1999, pp. 1226-1232.

[10] A. T. Hirsch, Z. J. Kaskal, C. W. Hertzer, et al., "ACC/
AHA 2005 Guidelines for the Management of Patients with Peripheral Arterial Disease (Lower Extremity, Renal, Mesenteric, and Abdominal Aortic): A Collaborative Report from the American Association for Vascular Surgery/Society for Vascular Surgery, Society for Cardiovascular Angiography and Interventions, Society for Vascular Medicine and Biology, Society of Interventional Radiology, and the ACC/AHA Task Force on Practice Guidelines (Writing Committee to Develop Guidelines for the Management of Patients with Peripheral Arterial Disease," Journal of the American College of Cardiology, Vol. 47, 2006, pp. 1-192. doi:10.1016/j.jacc.2006.02.024

[11] A. Alavi and E. J. Ring, "Localization of Gastrointestinal Bleeding: Superiority of 99 mTc Sulfur Colloid Compared with Angiography," American Journal of Roentgenology, Vol. 137, No. 4, 1981, pp. 741-748.

[12] D. A. Thorne, F. L. Datz, K. Remley, et al., "Bleeding Rates Necessary for Detecting Acute Gastrointestinal Bleeding with Technetium-99m-Labeled Red Blood Cells in an Experimental Model,” Journal of Nuclear Medicine, Vol. 28, No. 4, 1987, pp. 514-520.

[13] L. Defreyne, M. Uder, P. Vanlangenhove, et al., “Angiography for Acute Lower Gastrointestinal Hemorrhage: Efficacy of Cut Film Compared with Digital Subtraction Techniques," Journal of Vascular and Interventional Radiology, Vol. 14, No. 3, 2003, pp. 313-322. doi:10.1097/01.RVI.0000058414.01661.77

[14] K. H. Barth, "Radiological Intervention in Upper and Lower Gastrointestinal Bleeding,” Baillieres Clinical Gastroenterology, Vol. 9, No. 1, 1995, pp. 53-69. doi:10.1016/0950-3528(95)90070-5

[15] W. Browder, E. J. Cerise and M. S. Litwin, "Impact of Emergency Angiography in Massive Lower Gastrointestinal Bleeding,” Annals of Surgery, Vol. 204, No. 5, 1986, pp. 530-536. doi:10.1097/00000658-198611000-00004

[16] A. M. Vernava III, B. A. Moore, W. E. Longo, et al., "Lower Gastrointestinal Bleeding," Diseases of the Colon \& Rectum, Vol. 40, No. 7, 1997, pp. 846-858. doi:10.1007/BF02055445

[17] P. Orecchia, M. Hensley, P. Mcdonald, et al., "Localization of Lower Gastrointestinal Hemorrhage," Archives of Surgery, Vol. 120, No. 5, 1985, pp. 621-624. 